JOURNAL OF

FUNCTION SPACES AND APPLICATIONS

Volume 3, Number 3 (2005), 239-249
(C) 2005, Scientific Horizon http://www.jfsa.net

\title{
Matriceal Lebesgue spaces and Hölder inequality
}

\author{
Sorina Barza*, Dimitri Kravvaritis and Nicolae Popa ${ }^{\dagger}$ \\ (Communicated by Maria Carro)
}

2000 Mathematics Subject Classification. 42A16, 42A45, 47B35.

Keywords and phrases. Cesaro sums, Toeplitz matrices, Hilbert modules, Schur multipliers, Arveson projection.

Abstract. We introduce a class of spaces of infinite matrices similar to the class of Lebesgue spaces $L^{p}(\mathbb{T}), 1 \leq p \leq \infty$, and we prove matriceal versions of Hölder inequality.

\section{Introduction}

Let $A=\left(a_{i, j}\right)_{i, j \geq 1}$ be an infinite complex matrix. We denote by $A_{k}$, $k \in \mathbb{Z}$, the diagonal matrix whose entries $a_{i, j}^{\prime}$, satisfy the equation:

$$
a_{i, j}^{\prime}= \begin{cases}a_{i, j} & \text { if } j-i=k \\ 0 & \text { otherwise }\end{cases}
$$

\footnotetext{
${ }^{*}$ The research supported by: KAW 2000.0048 and EUROMMAT-ICA1-CT-20007022 .

${ }^{\dagger}$ The research supported by: EURROMMAT-ICA1-CT-2000-7022 and CERES 3-28.
} 
It was remarked first in [1] that there is a similarity between the Fourier series $\sum_{k \in \mathbb{Z}} a_{k} e^{i k t}$ of a function on the torus $\mathbb{T}=[0,2 \pi)$ and the decomposition $\sum_{k \in \mathbb{Z}} A_{k}$ of an infinite matrix in a sum of diagonal matrices.

Moreover Shields [8] observed the analogy between the convolution of two functions and the Schur product $A * B=\left(a_{i j} \cdot b_{i j}\right)_{i, j}$ of the matrices $A=\left(a_{i j}\right)_{i, j}$ and $B=\left(b_{i j}\right)_{i, j}$.

We denote by $B\left(\ell_{2}\right)$ the Banach space of all infinite matrices representing bounded operators acting on $\ell_{2}$ with respect to the standard basis, endowed with the operator norm. We denote also by $M\left(\ell_{2}\right)$ the space of all Schur multipliers with respect to the norm

$$
\|M\|_{M\left(\ell_{2}\right)}=\sup _{\|A\|_{B\left(\ell_{2}\right)} \leq 1}\|M * A\|_{B\left(\ell_{2}\right)}
$$

By reasons determined by Theorem 8.1 in [4] we will also call this space the space of matriceal measures.

A Toeplitz matrix $M$ is an infinite matrix with the entries $m_{i j}=m_{j-i}$ for all $i, j \geq 1$, where $\left(m_{j}\right)_{j}$ is a sequence of complex numbers. Sometimes we use for this matrix the notation $M=\left(m_{k}\right)_{k=-\infty}^{+\infty}$, and we denote by $\mathcal{T}$ the class of all infinite Toeplitz matrices.

Guided by the results in [4] and [10], which characterize the Toeplitz matrices in $M\left(\ell_{2}\right)$ and $B\left(\ell_{2}\right)$ by means of the associated Fourier series we extended in [3] some aspects of the classical Fejer's theory to the case of infinite matrices. We introduced there a space of continuous matrices $C\left(\ell_{2}\right)$ and a space of integrable matrices $L^{1}\left(\ell_{2}\right)$ and studied relations between them and the more classical $B\left(\ell_{2}\right)$ and $M\left(\ell_{2}\right)$ and also described some of their properties.

An important and well-known fact (see [10]) is the following: $B\left(l_{2}\right) \cap \mathcal{T}$ may be identified with $L^{\infty}(\mathbb{T})$ in the manner indicated in Theorem 0 , and we will write it rather abusive as $B\left(l_{2}\right) \cap \mathcal{T}=L^{\infty}(\mathbb{T})$. Similarly, $M\left(l_{2}\right) \cap \mathcal{T}=M(\mathbb{T})$ (see [4]) with equality of norms. Here of course, $M(\mathbb{T})$ is the space of all regular bounded Borel measures $\mu$ on $\mathbb{T}$, endowed with the norm

$$
\|\mu\|=|\mu|(\mathbb{T})
$$

where $|\mu|$ is the bounded variation of $\mu$.

So, we identify Toeplitz matrices with functions and we intend to extend some classical results from the Toeplitz matrices setting to the more general infinite matrices. 
Starting from these remarks, we consider, in the second section a class of matrix spaces similar to Lebesgue spaces $L^{p}(\mathbb{T}), 1 \leq p \leq \infty$, denoted by $L^{p}\left(l_{2}\right)$, such that $L^{p}\left(l_{2}\right) \cap \mathcal{T}=L^{p}(\mathbb{T}), 1 \leq p \leq \infty$.

We initiate also the study of the matriceal Hölder inequality. A naive extension of the classical Hölder inequality is, unfortunately, not valid.

We give some special classes of matrices, called matriceal Hardy spaces, such that this inequality will be true for them.

If $A=\left(a_{i j}\right)_{i, j \geq 1}$ we will denote by $A^{t}=\left(a_{j i}\right)_{i, j \geq 1}$ the transpose of $A$.

For every infinite matrix $A$, let us denote by $f_{A}=\sum_{k \in \mathbb{Z}} A_{k} e^{i k t}$. If $A=\left(a_{k}\right)_{k=-\infty}^{+\infty}$ is a Toeplitz matrix, then we get $f_{A}=\sum_{k \in \mathbb{Z}} a_{k} e^{i k t}$. Other notations used in the paper will be introduced as needed.

The following result is well-known: (See [10].)

Theorem 0. A Toeplitz matrix $A=\left(a_{k}\right)_{k=-\infty}^{+\infty}$ belongs to $B\left(\ell_{2}\right)$ if and only if the function $f_{A} \in L^{\infty}(\mathbb{T})$. Moreover

$$
\|A\|_{B\left(\ell_{2}\right)}=\left\|f_{A}\right\|_{L^{\infty}(\mathbb{T})} .
$$

We recall now that $A_{k}$ plays the role of the " $k^{\text {th }}$ Fourier coefficient of the matrix $A$." It is well-known that for each $f \in L^{\infty}(\mathbb{T})$ whose Fourier coefficients are $a_{n}, n \in \mathbb{Z}$, we have

$$
f \in C(\mathbb{T}) \text { if and only if } \lim _{n \rightarrow \infty}\left\|\sigma_{n}(f)-f\right\|_{L^{\infty}(\mathbb{T})}=0,
$$

where $\sigma_{n}(f)(t)=\sum_{k=-n}^{n}\left(1-\frac{|k|}{n+1}\right) a_{k} e^{i k t}$.

So, the following definition (see [3]) is natural:

Definition 1. Let $A \in B\left(\ell_{2}\right)$ and $\sigma_{n}(A)=\sum_{k=-n}^{n} A_{k}\left(1-\frac{|k|}{n+1}\right)$, $n=1,2, \ldots$, for $n \in \mathbb{N}^{*}$, the matriceal Fejer sum of the order $n$ associated to $A$.

Then we call a matrix $A$ to be a continuous matrix and we write $A \in C\left(\ell_{2}\right)$ if the following relation holds:

$$
\lim _{n \rightarrow \infty}\left\|\sigma_{n}(A)-A\right\|_{B\left(\ell_{2}\right)}=0 .
$$

Obviously $C\left(\ell_{2}\right)$ endowed with the operator norm becomes a Banach space.

Theorem 0 allows us to write the formula

$$
\left[B\left(\ell_{2}\right) \cap \mathcal{T}\right]^{*}=L^{\infty}(\mathbb{T}),
$$


where by $[H]^{*}$ we denote the image of the space $H$ of matrices by the correspondence $A \rightarrow f_{A}$.

Remark 2. For brevity we write in what follows equations like the previous one in the following manner:

$$
\begin{aligned}
& B\left(\ell_{2}\right) \cap \mathcal{T}=L^{\infty}(\mathbb{T}), \\
& C\left(\ell_{2}\right) \cap \mathcal{T}=C(\mathbb{T}) .
\end{aligned}
$$

In the sequel we use another notation, more appropriate to our aims, for the entries of the matrix $A$, namely we put

$$
a_{k}^{l}=\left\{\begin{array}{ll}
a_{l, l+k} & k \geq 0, l=1,2,3 \ldots \\
a_{l-k, l} & k<0, l=1,2,3, \ldots
\end{array},\right.
$$

and denote $A$ sometimes as $A=\left(a_{k}^{l}\right)_{k \in \mathbb{Z}, l \geq 1}$.

Let $A^{(l)}=\left(b_{k}^{m}\right)_{k \in \mathbb{Z}, m \geq 1}$, where $l \in \mathbb{N}^{*}$, be the matrix given by

$$
b_{k}^{m}= \begin{cases}a_{k}^{l} & \text { if } m=l \\ 0 & \text { if } m \neq l\end{cases}
$$

We call the matrix $A^{(l)}$, the $l^{\text {th }}$-corner matrix associated to $A$.

Now, if for any corner-matrix $A^{(l)}=\left(b_{k}^{m}\right)_{k \in Z, m \geq 1}$ we associate a distribution on $\mathbb{T}$, denoted by $f_{l}$ such that $b_{k}^{l}=\widehat{f}_{l}(k)$, we get, in case $A \in B\left(\ell_{2}\right) \cap \mathcal{T}$, that $f_{l}=f \in L^{\infty}(\mathbb{T})$, for all $l \in \mathbb{N}^{*}$.

We can identify the matrix $A=\left(A^{(l)}\right)_{l \in \mathbb{N}^{*}}$ with its sequence of associated distributions $\mathbf{f} \stackrel{\text { not }}{=}\left(f_{l}\right)_{l \in \mathbb{N}^{*}}$, writing this fact as

$$
A=A_{\mathbf{f}}
$$

By Theorem 0 we have the following correspondences:

$$
\begin{aligned}
& f \in L^{\infty}(\mathbb{T}) \text { if and only if } A_{\mathbf{f}} \in B\left(\ell_{2}\right) \cap \mathcal{T}, \text { where } \mathbf{f}=(f, f, f, \ldots) \\
& g \in L^{\infty}(\mathbb{T}) \text { if and only if } A_{\mathbf{g}} \in B\left(\ell_{2}\right) \cap \mathcal{T}, \text { where } \mathbf{g}=(g, g, g, \ldots) .
\end{aligned}
$$

Then, of course, it follows

$$
f g \in L^{\infty}(\mathbb{T}) \text { if and only if } A_{\mathbf{f g}} \in B\left(\ell_{2}\right) \cap \mathcal{T}, \text { where } \mathbf{f g}=(f g, f g, f g, \ldots) \text {. }
$$

The matrix $A=\left(a_{i j}\right)$ is said to be of $n$-band type if $a_{i j}=0$ for $|i-j|>n$.

Having these notions in mind we introduce a commutative product of infinite matrices: 
Definition 3. Let $A=A_{\mathbf{f}}$ and $B=A_{\mathbf{g}}$ two infinite matrices of finite band type. We introduce now the commutative product $\square$ given by

$$
A \square B \stackrel{\text { def }}{=} A_{\mathrm{fg}} .
$$

Remark 4. (1) We mention that in the previous definition we took $A=A_{\mathbf{f}}, B=A_{\mathbf{g}}$ infinite matrices of finite band type since, $f$ and $g$ being trigonometric polynomials, we may consider the product $f g$.

(2) This product can be defined also for all matrices $A, B \in B\left(\ell_{2}\right)$, but $A \square B$ does not belong in general to $B\left(\ell_{2}\right)$. Take, for instance, $A=A_{\mathbf{f}}$, with $\mathbf{f}=(f, 0,0, \ldots)$, and $B=A_{\mathbf{g}}$, with $\mathbf{g}=(g, 0,0, \ldots)$, where $f, g \in H^{2}(\mathbb{T})$ such that $f g \in H^{1}(\mathbb{T}) \backslash H^{2}(\mathbb{T})$.

(3) Of course, if $A_{\mathbf{f}}, A_{\mathbf{g}} \in B\left(\ell_{2}\right) \cap \mathcal{T}$ then it follows that $A_{\mathbf{f}} \square A_{\mathbf{g}}=A_{\text {fg }} \in$ $B\left(\ell_{2}\right) \cap \mathcal{T}$.

\section{Lebesgue spaces of infinite matrices and Hölder inequality}

We intend now to introduce some spaces of infinite matrices which are similar to classical Lebesgue spaces $L^{p}(\mathbb{T}), 1 \leq p \leq \infty$.

We recall here the following: (See [3]. )

Definition 5. Let $L^{1}\left(\ell_{2}\right)=\left\{A \in M\left(\ell_{2}\right)\right.$ such that $\lim _{n \rightarrow \infty}\left\|\sigma_{n}(A)-A\right\|_{M\left(\ell_{2}\right)}$ $=0\}$. We consider on $L^{1}\left(\ell_{2}\right)$ the induced norm of $M\left(\ell_{2}\right)$.

We need also a space of infinite matrices which is similar to Hilbert space $L^{2}(\mathbb{T})$ and, in fact, extends it.

Let first consider the space

$$
\mathcal{L}^{2}\left(\ell_{2}\right) \stackrel{\text { def }}{=}\left\{\begin{array}{c}
A \text { infinite matrices such that } P_{T} A \in B\left(\ell_{2}, \ell_{\infty}\right) \text { and } \\
{\left[\left(I-P_{T}\right)(A)\right]^{t} \in B\left(\ell_{2}, \ell_{\infty}\right)}
\end{array}\right\},
$$

where $I$ is the identity operator,

$$
P_{T}(A)=\left\{\begin{array}{cc}
a_{i, j} & \text { if } i \leq j \\
0 & \text { otherwise }
\end{array}\right.
$$

and $B\left(\ell_{2}, \ell_{\infty}\right)$ is the space of all matrices representing bounded linear operators from $\ell_{2}$ into $\ell_{\infty}$, endowed with operator norm.

On $\mathcal{L}^{2}\left(\ell_{2}\right)$ we introduce the norm $\|A\|_{\mathcal{L}^{2}\left(\ell_{2}\right)}=\sup _{l \in \mathbb{Z}}\left(\sum_{j=1}^{\infty}\left|a_{l}^{j}\right|^{2}\right)^{\frac{1}{2}}$. 
Motivated by Definitions 1 and 5 we consider the space $L^{2}\left(\ell_{2}\right) \stackrel{\text { def }}{=}\left\{A \in \mathcal{L}^{2}\left(\ell_{2}\right)\right.$ such that $\lim _{n \rightarrow \infty} \sigma_{n}(A)=A$ in the norm of $\left.\mathcal{L}^{2}\left(\ell_{2}\right)\right\}$.

It is easy to see that

$$
L^{2}\left(\ell_{2}\right) \subset L^{1}\left(\ell_{2}\right)
$$

and also $L^{2}\left(\ell_{2}\right) \cap \mathcal{T}=L^{2}(\mathbb{T})$.

$L^{2}\left(\ell_{2}\right)$ can be regarded as a matriceal extension of the familiar Hilbert space $L^{2}(\mathbb{T})$.

A good reason for this is the fact that we may introduce in $L^{2}\left(\ell_{2}\right)$ an extension of the usual scalar product.

We consider now the Arveson projection (see [2]) $P: B\left(\ell_{2}\right) \rightarrow B\left(\ell_{2}\right) \cap \mathcal{T}$ given by a fixed Banach limit $\Lambda$. More precisely, for $A=\left(a_{j}^{l}\right)_{j \in \mathbb{Z}, l \geq 1}$ and $a_{j}=\left(a_{j}^{l}\right)_{l \geq 1}$, we put $P(A)=\left(\Lambda\left(a_{j}\right)\right)_{j=-\infty}^{+\infty}$.

It is easy to see, using the definitions, that:

Theorem 6. The Arveson projection associated to some Banach limit $\Lambda, P(A)=\sum_{j \in \mathbb{Z}} \Lambda\left(a_{j}\right)$, where $A=\left(a_{j}^{l}\right)_{j \in \mathbb{Z}, l \geq 1}$, maps continuously $C\left(\ell_{2}\right)$ onto $C\left(\ell_{2}\right) \cap \mathcal{T}$, and moreover, $\|P\| \leq\|\Lambda\|$.

Now we give the analogue of Theorem 6 for $L^{1}\left(\ell_{2}\right)$.

Theorem 7. Let $P$ be the Arveson projection. Then $P: M\left(\ell_{2}\right) \rightarrow$ $M\left(\ell_{2}\right) \cap \mathcal{T}$ and $P: L^{1}\left(\ell_{2}\right) \rightarrow L^{1}\left(\ell_{2}\right) \cap \mathcal{T}$ are continuous linear maps with $\|P\| \leq\|\Lambda\|$.

Proof. Let $A=\left(a_{j}^{l}\right)_{j \in \mathbb{Z}, l \geq 1}$. We have:

$$
\begin{aligned}
\|P(A)\|_{M\left(\ell_{2}\right)} & =\left\|\sum_{k=-\infty}^{\infty} \Lambda\left(a_{k}\right) e^{i k t}\right\|_{M(\mathbb{T})} \\
& =\text { (by Theorem 16.3.5, [7]) } \\
& =\sup _{\|g\|_{L^{\infty} \leq 1} \|} \sum_{k=-\infty}^{+\infty} \Lambda\left(a_{j}\right) e^{i k t} * g \|_{L^{\infty}(\mathbb{T})} \\
& =\sup _{B B \| 1}\|P(A) * B\|_{B\left(\ell_{2}\right)} \\
& =\sup _{\|B\| \leq 1, B \in \mathcal{T}}\|P(A * B)\|_{B\left(\ell_{2}\right)} \\
& \leq(\text { by }[2]) \leq\|\Lambda\| \cdot \sup _{\|B\| \leq 1}\|A * B\|_{B\left(\ell_{2}\right)} \\
& =\|\Lambda\| \cdot\|A\|_{M\left(\ell_{2}\right)} \cdot
\end{aligned}
$$


Hence $P: M\left(\ell_{2}\right) \rightarrow M(\mathbb{T})$ is a bounded projection with $\|P\| \leq\|\Lambda\|$. By Definition 5 this proves also the assertion concerning $L^{1}\left(\ell_{2}\right)$.

Obviously $C\left(\ell_{2}\right) \varsubsetneqq B\left(\ell_{2}\right) \varsubsetneqq L^{2}\left(\ell_{2}\right) \varsubsetneqq L^{1}\left(\ell_{2}\right)$.

It is also easy to see that:

Theorem 8. The Arveson projection $P$, given by a fixed Banach limit $\Lambda$, maps continuously $L^{2}\left(\ell_{2}\right)$ onto $L^{2}\left(\ell_{2}\right) \cap \mathcal{T}$ and $\|P\| \leq\|\Lambda\|$.

So, by Theorem 6 , it follows that $C\left(\ell_{2}\right) \cap \mathcal{T}=C(\mathbb{T})$ is a complemented subspace of $C\left(\ell_{2}\right)$ with respect to the Arveson projection $P$ and by Theorem 8 the space $L^{2}\left(\ell_{2}\right) \cap \mathcal{T}=L^{2}(\mathbb{T})$ is a complemented subspace of $L^{2}\left(\ell_{2}\right)$.

We may consider now the complex interpolation space (see [6], [9]) $\left[C\left(\ell_{2}\right), L^{2}\left(\ell_{2}\right)\right]_{\frac{2}{p}}$ and call it the matriceal Lebesgue space $L^{p}\left(\ell_{2}\right)$, for $2 \leq p \leq \infty$.

Now we can use a general result of interpolation theory (See [9], 1.17.1, Theorem 1.)

Theorem A. Let $\left(A_{0}, A_{1}\right)$ be an interpolation pair of Banach spaces and $B$ a complemented subspace of $A_{0}+A_{1}$, such that the corresponding projection $P$ maps continuously $A_{0}$ into $A_{0}$ and $A_{1}$ into $A_{1}$. Let $F$ be an interpolation functor. Then $\left(A_{0} \cap B, A_{1} \cap B\right)$ is an interpolation pair and

$$
F\left(A_{0} \cap B, A_{1} \cap B\right)=F\left(A_{0}, A_{1}\right) \cap B .
$$

Indeed we put in Theorem A $A_{0}=B\left(\ell_{2}\right), A_{1}=L^{2}\left(\ell_{2}\right), B=$ $L^{2}\left(\ell_{2}\right) \cap \mathcal{T}=L^{2}(\mathbb{T}), \quad P: L^{2}\left(\ell_{2}\right) \rightarrow L^{2}(\mathbb{T})$ the Arveson projection and $F=\left[C\left(\ell_{2}\right), L^{2}\left(\ell_{2}\right)\right]_{\frac{2}{p}}$, for $2 \leq p \leq \infty$. Then, by Theorems 6 and $8, B$ and $P$ satisfy the conditions of Theorem $\mathrm{A}$, hence $\left(C(\mathbb{T}), L^{2}(\mathbb{T})\right)$ is an interpolation pair and

$$
L^{p}(\mathbb{T})=\left[C(\mathbb{T}), L^{2}(\mathbb{T})\right]_{\frac{2}{p}}=\left[C\left(\ell_{2}\right), L^{2}\left(\ell_{2}\right)\right]_{\frac{2}{p}} \cap \mathcal{T}=L^{p}\left(\ell_{2}\right) \cap \mathcal{T}
$$

Similarly, using Theorems 7 and 8 in Theorem A, we get

$$
L^{p}(\mathbb{T})=\left[L^{2}(\mathbb{T}), L^{1}(\mathbb{T})\right]_{\frac{2-p}{p}}=\left[L^{2}\left(\ell_{2}\right), L^{1}\left(\ell_{2}\right)\right]_{\frac{2-p}{p}} \cap \mathcal{T}=L^{p}\left(\ell_{2}\right) \cap \mathcal{T},
$$

for $1 \leq p \leq 2$.

Here, of course, we denote $\left[L^{2}\left(\ell_{2}\right), L^{1}\left(\ell_{2}\right)\right]_{\frac{2-p}{p}}$ by $L^{p}\left(\ell_{2}\right)$, for $1 \leq p \leq 2$.

In order to justify the introduction of above matrix spaces we would like to prove a version of Hölder inequality in a subspace of $L^{p}\left(\ell_{2}\right)$, for $2 \leq p \leq \infty$. 
Definition 9. We denote by $H^{1}\left(\ell_{2}\right)=\left\{A \in L^{1}\left(\ell_{2}\right) ; A\right.$ upper triangular $\}$ and we call $H^{1}\left(\ell_{2}\right)$ the matriceal Hardy space of order 1 .

Then $H^{1}\left(\ell_{2}\right)$ is a Banach subspace of $L^{1}\left(\ell_{2}\right)$ and $A \in H^{1}\left(\ell_{2}\right) \cap \mathcal{T}$ if and only if $f_{A} \in H^{1}(\mathbb{T})$.

Similarly $H^{\infty}\left(\ell_{2}\right)=\left\{A \in B\left(\ell_{2}\right) ; A\right.$ upper triangular $\}$. Of course $H^{\infty}\left(\ell_{2}\right)$ is a Banach subspace of $B\left(\ell_{2}\right)$ and $A \in H^{\infty}\left(\ell_{2}\right) \cap \mathcal{T}$ if and only if $f_{A} \in H^{\infty}(\mathbb{T})$.

In what follows we consider only upper triangular matrices.

Then, by straightforward computation, we get:

Remark 10. Let $A \in \mathcal{T}$ such that $f_{A}(t)$ be an analytic polynomial $P_{n}\left(e^{i t}\right)$ and $B$ an upper triangular matrix. Then

$$
A \square B=B \cdot A,
$$

where $A \cdot B$ means the usual non commutative product of matrices.

Now we are interested to find a matriceal version of the well-known Hölder inequality.

At the first glance we are tempted to look at the following naïve matriceal Hölder inequality in case $p=1, q=\infty$.

$$
A \in B\left(\ell_{2}\right) \text { and } B \in L^{1}\left(\ell_{2}\right) \text { implies } A \square B \in L^{1}\left(\ell_{2}\right) .
$$

Unfortunately the previous statement does not hold. For instance:

Example 11. Let $A=(f, 0,0, \ldots) \in B\left(\ell_{2}\right)$, where $f(t)=1+\sum_{k=1}^{\infty} \frac{1}{k} \cos k t$ and let $B=(g, 0,0, \ldots) \in L^{1}\left(\ell_{2}\right)$, for $g(t)=\sum_{k=1}^{\infty} \frac{1}{\ln k} e^{i k t}$.

Then it follows easily that $(A \square B)_{0}^{1}=\sum_{k=2}^{\infty} \frac{1}{k \ln k}=\infty$.

However, clearly if $A \in B\left(\ell_{2}\right) \cap \mathcal{T}$ and if $B \in L^{1}\left(\ell_{2}\right) \cap \mathcal{T}$ then it follows that $A \square B \in L^{1}\left(\ell_{2}\right) \cap \mathcal{T}$.

Of course there are also other classes of matrices $A, B$ such that $A \square B \in L^{1}\left(\ell_{2}\right)$.

Theorem 12. Let $A$ and $B$ upper triangular matrices such that $A \in \mathcal{T}$ with $f_{A} \in A(D)$, and $B \in H^{1}\left(\ell_{2}\right)$. Then $A \square B \in H^{1}\left(\ell_{2}\right)$ and

$$
\|A \square B\|_{H^{1}\left(\ell_{2}\right)} \leq\|B\|_{H^{1}\left(\ell_{2}\right)} \cdot\left\|f_{A}\right\|_{A(D)} .
$$


Proof. It is enough to prove theorem in the case $f_{A}$ equal to the analytic polynomial $P_{n}(t)=\sum_{k=0}^{n} a_{k} e^{i k t}$.

Then, for any $C \in B\left(\ell_{2}\right)$, we get, using Remark 10,

$$
(A \square B) * C=(B \cdot A) * C=B *\left(a_{0} C+a_{1} C_{1}+\cdots+a_{n} C_{n}\right),
$$

where

$$
\begin{aligned}
C_{1} & =\left(\begin{array}{lllll}
0 & c_{11} & \ldots & c_{1 n} & \ldots \\
0 & c_{21} & \ldots & c_{2 n} & \ldots \\
\vdots & \vdots & \vdots & \vdots & \vdots
\end{array}\right), \ldots \\
C_{n} & =\left(\begin{array}{llllll}
0 & \ldots & 0 & c_{11} & c_{12} & \ldots \\
0 & \ldots & 0 & c_{21} & c_{22} & \ldots \\
\vdots & \vdots & \vdots & \vdots & \vdots & \vdots
\end{array}\right) .
\end{aligned}
$$

Thus

$$
\begin{gathered}
\|(A \square B) * C\|_{B\left(\ell_{2}\right)}=\left\|B *\left(a_{0} C+a_{1} C_{1}+\cdots+a_{n} C_{n}\right)\right\|_{B\left(\ell_{2}\right)} \leq\|B\|_{H^{1}\left(\ell_{2}\right)} . \\
\left\|a_{0} C+a_{1} C_{1}+\cdots+a_{n} C_{n}\right\|_{B\left(\ell_{2}\right)} .
\end{gathered}
$$

But, defining by $\tau_{-k}: \ell_{2}(\mathbb{N}) \rightarrow \ell_{2}(\mathbb{N})$ for $k \in \mathbb{N}$, the operator defined by $\tau_{-k}\left(x_{0}, x_{1}, x_{2}, \ldots\right)=\left(x_{k}, x_{k+1}, \ldots\right)$ for all $x=\left(x_{0}, x_{1}, \ldots\right) \in \ell_{2}(\mathbb{N})$, we get

$$
\begin{aligned}
\left\|a_{0} C+a_{1} C_{1}+\ldots+a_{n} C_{n}\right\|_{B\left(\ell_{2}\right)} & =\sup _{\|x\|_{2} \leq 1}\left\|C\left(a_{0} x+a_{1} \tau_{-1} x+\ldots+a_{n} \tau_{-n} x\right)\right\|_{2} \\
& =\sup _{\|x\|_{2} \leq 1}\left\|C\left(a_{0} E_{0}+a_{1} E_{1}+\ldots+a_{n} E_{n}\right) x\right\|_{2} \\
& =\left\|C\left(\sum_{k=0}^{n} a_{k} E_{k}\right)\right\|_{B\left(\ell_{2}\right)} \\
& \leq\|C\|_{B\left(\ell_{2}\right)} \cdot\left\|P_{n}\right\|_{C(\mathbb{T})} .
\end{aligned}
$$

(Here, of course, $E_{k}$ is the $k^{\text {th }}$-diagonal matrix associated to $E$ whose entries are all equal to 1.)

Now

$$
\begin{aligned}
\|A \square B\|_{H^{1}\left(\ell_{2}\right)} & =\sup _{\|C\|_{B\left(\ell_{2}\right)} \leq 1}\|(A \square B) * C\|_{B\left(\ell_{2}\right)} \\
& \leq\|B\|_{H^{1}\left(\ell_{2}\right)} \cdot\left\|f_{A}\right\|_{A(D)} .
\end{aligned}
$$


We define now the space

$H^{2}\left(\ell_{2}\right) \stackrel{\text { def }}{=}\left\{A\right.$ upper triangular matrix ; $\left.\lim _{n \rightarrow \infty}\left\|\sigma_{n}(A)-A\right\|_{B\left(\ell_{2}, \ell_{\infty}\right)}=0\right\}$.

Of course $A \in H^{2}\left(\ell_{2}\right) \cap \mathcal{T}$ if and only if $f_{A} \in H^{2}(\mathbb{T})$.

Then we get

Theorem 13. (Matriceal Cauchy-Schwarz inequality) Let $A \in \mathcal{T}$ such that $f_{A} \in H^{2}(\mathbb{T})$ and $B \in H^{2}\left(\ell_{2}\right)$. Then $A \square B \in H^{1}\left(\ell_{2}\right)$ and

$$
\|A \square B\|_{H^{1}\left(\ell_{2}\right)} \leq\left\|f_{A}\right\|_{H^{2}(\mathbb{T})} \cdot\|B\|_{H^{2}\left(\ell_{2}\right)} .
$$

Proof. Since $\|A \cdot B\|_{M\left(\ell_{2}\right)} \leq\|A\|_{B\left(\ell_{2}, \ell_{\infty}\right)} \cdot\|B\|_{B\left(\ell_{1}, \ell_{2}\right)}$ (see Theorem 6.4, $[4])$, if $f_{A}=P_{n}$, we get that $\|A \square B\|_{M\left(\ell_{2}\right)}=\|B \cdot A\|_{M\left(\ell_{2}\right)} \leq\|B\|_{B\left(\ell_{2}, \ell_{\infty}\right)}$. $\|A\|_{B\left(\ell_{1}, \ell_{2}\right)}=\left\|f_{A}\right\|_{H^{2}(\mathbb{T})} \cdot\|B\|_{H^{2}\left(\ell_{2}\right)}$, which proves the theorem.

In order to state a more general Hölder inequality for matrices we use the complex interpolation for spaces of upper triangular matrices; more precisely we consider the spaces $\left[A(D), H^{2}(\mathbb{T})\right]_{\frac{2}{p}}=H^{p}(\mathbb{T})$, (see $\left.[5]\right)$ and $\left[H^{1}\left(\ell_{2}\right), H^{2}\left(\ell_{2}\right)\right]_{\frac{2}{p}}$, for $2 \leq p \leq \infty$.

By Theorems 7 and 8 it follows that Arveson projection maps $H^{1}\left(\ell_{2}\right)$ onto $H^{1}\left(\ell_{2}\right) \cap \mathcal{T}=H^{1}(\mathbb{T}), H^{2}\left(\ell_{2}\right)$ onto $H^{2}\left(\ell_{2}\right) \cap \mathcal{T}=H^{2}(\mathbb{T})$. On the other side $\left[H^{1}\left(\ell_{2}\right), H^{2}\left(\ell_{2}\right)\right]_{\frac{2}{p}}=\left[H^{2}\left(\ell_{2}\right), H^{1}\left(\ell_{2}\right)\right]_{\frac{p-2}{p}}$ and the last space will be denoted by $H^{q}\left(\ell_{2}\right)$, where $p^{-1}+q^{-1}=1$. The reason for this last notation is that $H^{q}\left(\ell_{2}\right) \cap \mathcal{T}=H^{q}(\mathbb{T})$, since, by Theorem A, it is clear that $\left[H^{2}(\mathbb{T}), H^{1}(\mathbb{T})\right]_{\theta}=\left[H^{2}\left(\ell_{2}\right) \cap \mathcal{T}, H^{1}\left(\ell_{2}\right) \cap \mathcal{T}\right]_{\theta}=\left[H^{2}\left(\ell_{2}\right), H^{1}\left(\ell_{2}\right)\right]_{\theta} \cap \mathcal{T}$, for $0<\theta<1$.

We consider the complex interpolation norm on $H^{q}\left(\ell_{2}\right)$ and we call this space the matriceal Hardy space of order $q$.

Since $H^{1}\left(\ell_{2}\right)$ is not complemented in $L^{1}\left(\ell_{2}\right)$ we ask ourselves:

Problem. Is it $H^{q}\left(\ell_{2}\right)$ a Banach subspace of $L^{q}\left(\ell_{2}\right)$ ?

Of course $H^{q}\left(\ell_{2}\right) \cap \mathcal{T}=H^{q}(\mathbb{T})$ is a Banach subspace of $L^{q}\left(\ell_{2}\right) \cap \mathcal{T}=L^{q}(\mathbb{T})$ and $H^{q}\left(\ell_{2}\right)$ is a linear subspace of $L^{q}\left(\ell_{2}\right)$, for all $1 \leq q \leq \infty$.

Using the well-known result about complex interpolation of bilinear maps (see [6]-10.1) and theorems 12 and 13 we get a matriceal version of classical Hölder inequality for analytic functions:

Theorem 14. Let $A \in \mathcal{T}$ such that $f_{A} \in H^{p}(\mathbb{T})$, for $2 \leq p \leq \infty$, and $B \in H^{q}\left(\ell_{2}\right)$, for $p^{-1}+q^{-1}=1$. Then $A \square B \in H^{1}\left(\ell_{2}\right)$ and

$$
\|A \square B\|_{H^{1}\left(\ell_{2}\right)} \leq\left\|f_{A}\right\|_{H^{p}(\mathbb{T})} \cdot\|B\|_{H^{q}\left(\ell_{2}\right)} .
$$




\section{References}

[1] J. Arazy, Some remarks on interpolation theorems and the boundness of the triangular projection in unitary matrix spaces, Integral Equations Operator Theory, 1 (1978), 453-495.

[2] W. Arveson, Interpolation problems in nest algebras, J. Funct. Anal., 20 (1975), 208-253.

[3] S. Barza, L.-E. Persson and N. Popa, A matriceal analogue of Fejer's theory, Math. Nachr., 260 (2003), 14-20.

[4] G. Bennett, Schur multipliers, Duke Math. J., 44 (1977), 603-639.

[5] C. Bennet and R. Sharpley, Interpolation of operators, Academic Press, Boston, 1988.

[6] A. P. Calderon, Intermediate spaces and interpolation, the complex method, Studia Math., 24 (1964), 113-190.

[7] R. E. Edwards, Fourier Series, A Modern Introduction, Vol. I \& II, 2nd edn., Springer Verlag, Berlin, 1979, 1982.

[8] A. L. Shields, An analogue of a Hardy-Littlewood-Fejer inequality for upper triangular trace class operators, Math. Z., 182 (1983), 473-484.

[9] H. Triebel, Interpolation theory, function spaces, differential operators, VEB Deutscher Verlag der Wissenschaften, Berlin, 1978.

[10] K. Zhu, Operator Theory in Banach Function Spaces, Marcel Dekker, New York, 1990.

Department of Eng. Sciences

Physics and Mathematics, University of Karlstad

S-651 88 Karlstad

Sweden

(E-mail : sorina.barza@kau.se)

Department of Mathematics

National Technical University of Athens

15780 Zografou-Campus Athens

Greece

(E-mail : dkrav@math.ntua.gr)

Inst. of Mathematics, Romanian Academy

Box 1-764 70700

Bucharest

Romania

(E-mail : npopa@imar.ro)

(Received : September 2004) 


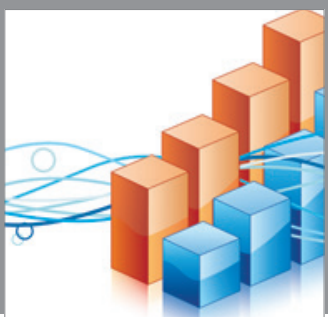

Advances in

Operations Research

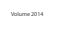

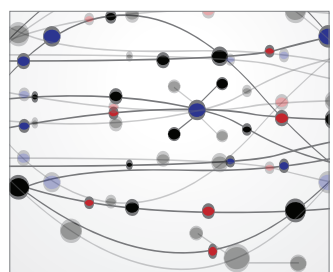

\section{The Scientific} World Journal
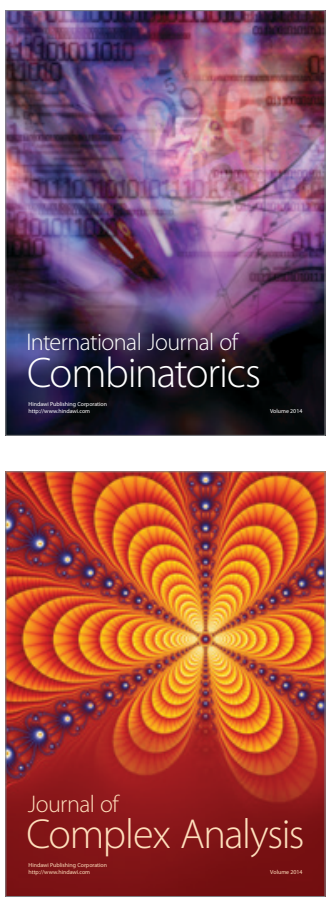

International Journal of

Mathematics and

Mathematical

Sciences
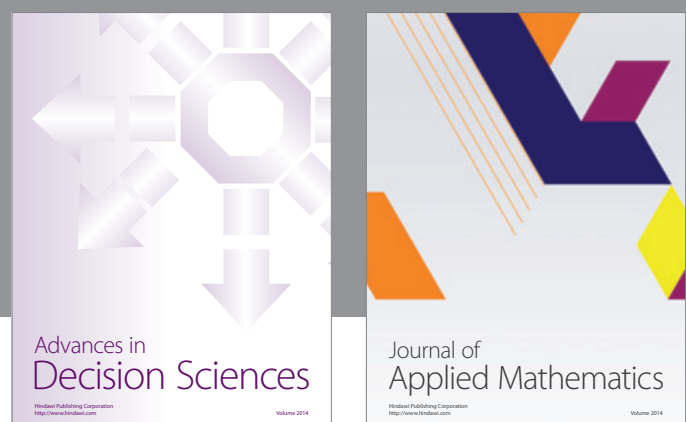

Journal of

Applied Mathematics
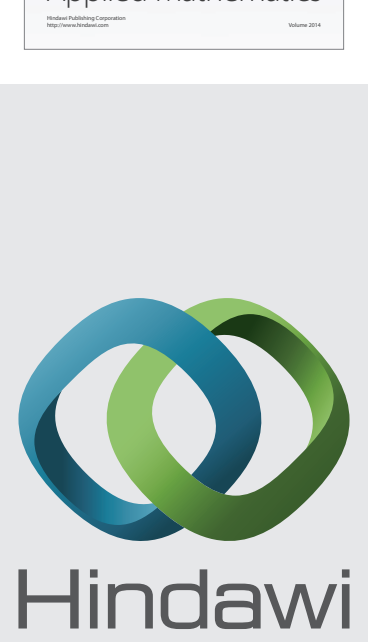

Submit your manuscripts at http://www.hindawi.com
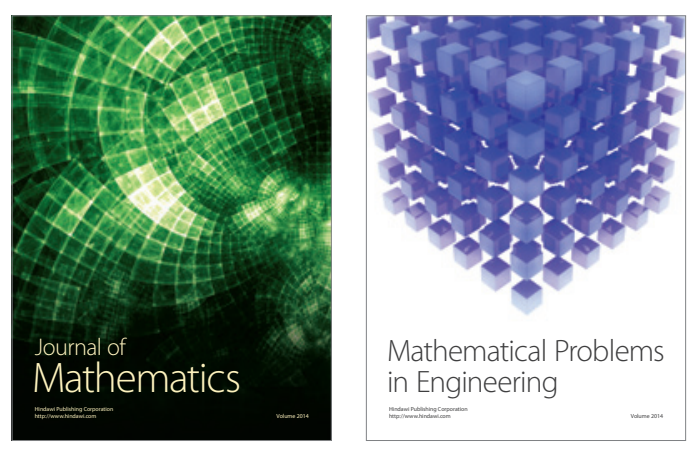

Mathematical Problems in Engineering
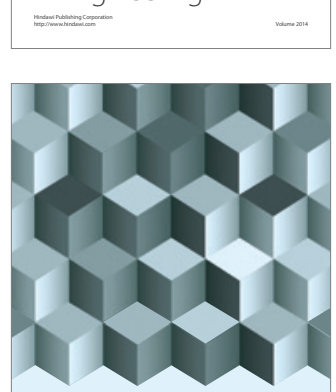

Journal of

Function Spaces
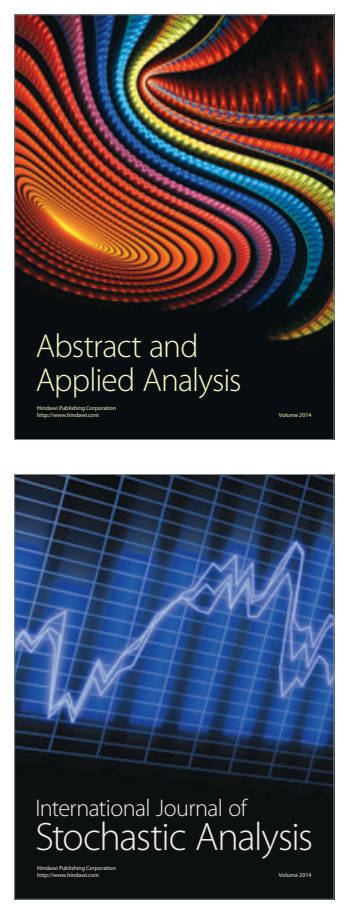

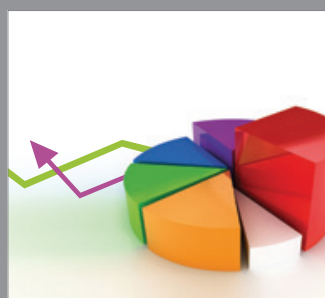

ournal of

Probability and Statistics

Promensencen
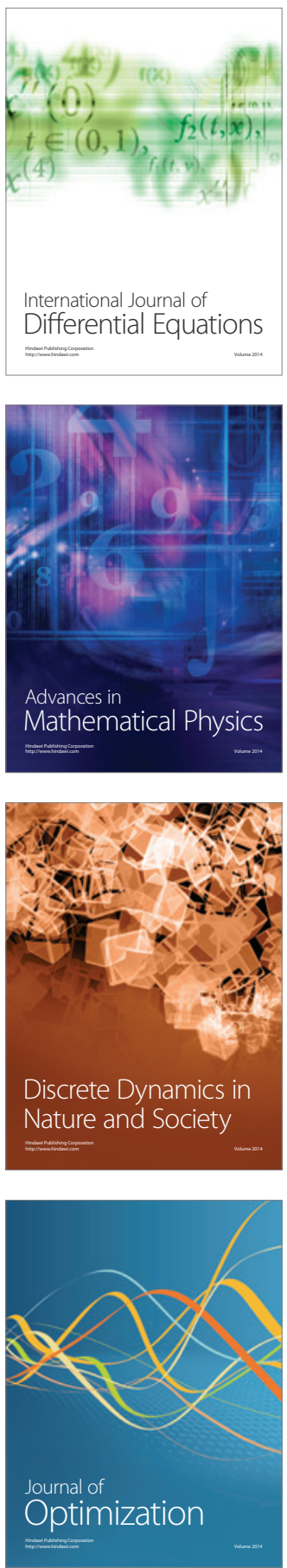